\title{
A Case of Carcinoma Showing Thymus-Like Differentiation with a Rapidly Lethal Course
}

\author{
Tomohiro Nogami $^{\mathrm{a}} \quad$ Naruto Taira $^{\mathrm{a}}$ Shinichi Toyooka ${ }^{\mathrm{b}}$ \\ Takehiro Tanaka $^{c}$ Taeko Mizoo $^{a}$ Takayuki Iwamoto ${ }^{a}$ Tadahiko Shien ${ }^{a}$ \\ Junichi Soh $^{\mathrm{b}}$ Shinichiro Miyoshi ${ }^{\mathrm{b}}$ Hiroyoshi Doihara ${ }^{\mathrm{a}}$ \\ Departments of ${ }^{\mathrm{a}}$ Breast and Endocrine Surgery, ${ }^{\mathrm{b}}$ Thoracic Surgery and ${ }^{\mathrm{C}}$ Pathology, \\ Okayama University Hospital, Okayama, Japan
}

\section{Key Words}

Carcinoma showing thymus-like differentiation $\cdot$ Infiltrating trachea $\cdot$ Reconstruction

\begin{abstract}
A 55-year-old woman underwent a total thyroidectomy for carcinoma showing thymus-like differentiation (CASTLE). The patient was referred to our hospital after the tumor was found to have directly invaded the cervical esophagus and the entire circumference of the trachea. A total thyroidectomy was performed, followed by end-to-end anastomosis of the trachea, suprahyoid release and dissection of bilateral pulmonary ligaments. No major complications, including anastomotic dehiscence or stenosis, were observed. The patient experienced some swallowing disturbances and hoarseness during the perioperative period but fully recovered. Radiotherapy to the neck was performed as an adjuvant therapy. Eleven months after surgery, lower back pain and right leg numbness developed and led to gait inability. Multiple lung and bone recurrences were observed, but no local recurrence. Palliative radiotherapy to the bone metastasis was performed. The patient died of pleural metastasis 14 months after the initial diagnosis of CASTLE.

(C) 2014 S. Karger AG, Basel
\end{abstract}

\section{Introduction}

Carcinoma showing thymus-like differentiation (CASTLE) is a rare thyroid tumor that resembles squamous cell carcinoma (SCC) of the thymus. This disease was first reported by Miyauchi et al. [1] in 1985 as intrathyroidal epithelial thymoma and classified by Chan and

Tomohiro Nogami

Department of Breast and Endocrine Surgery

Okayama University Hospital

2-5-1 Kita-ku, Shikata-cho, Okayama-city, Okayama 700-8558 (Japan)

E-Mail imagon12000@yahoo.co.jp 
Nogami et al:: A Case of Carcinoma Showing Thymus-Like Differentiation with a Rapidly Lethal Course

Rosai [2] in 1991. CASTLE often occurs in adults in their 50s and is considered to originate from ectopic thymic tissue or remnants related to thymic development or the thyroid, because the tumor usually occurs in the lower part of the thyroid gland and exhibits several features of thymic differentiation [3]. CASTLE frequently infiltrates adjacent soft tissue and metastasizes to regional lymph nodes, but patients with CASTLE typically have a favorable prognosis $[3,4]$. Thus, a differential diagnosis should include consideration of primary SCC of the thyroid, undifferentiated carcinoma with squamous features, and metastatic SCC from other organs, since CASTLE may histologically resemble these diseases and may even include foci of squamous differentiation [4]. We report here a case of CASTLE that had infiltrated the trachea. The tumor was resected, and tracheal mobilization and direct anastomosis of the trachea were performed. The patient's bilateral vocal functions were almost completely restored.

\section{Case Report}

A 52-year-old woman was referred to our hospital because of severe dyspnea and the presence of a neck tumor. She had experienced dyspnea for the past 6 months and had recently noticed bloody sputum. A computed tomography examination of the neck revealed a thyroid tumor about $8 \mathrm{~cm}$ in size. The tumor had invaded the trachea, obstructing $90 \%$ of the tracheal lumen (fig. 1a). An emergency tracheotomy was performed to maintain an airway through the tumor tissue. On biopsy, the tumor was diagnosed as CASTLE, suggesting a favorable prognosis if a complete resection could be performed. Magnetic resonance imaging and an esophageal fibroscopy examination revealed a low possibility of esophageal invasion (fig. 1b). Under general anesthesia, a thyroidectomy was initially performed via a neck incision. The invaded portion of the trachea was sharply dissected. A partial sternotomy was performed at the level of the 2nd intercostal space to identify the distal portion of the intact trachea and to mobilize the inferior tracheal segment. The trachea was transversely opened to find the intact portion. The length of the resected trachea was $6 \mathrm{~cm}$ (fig. 2). Bilateral recurrent nerves were macroscopically preserved. We performed a suprahyoid release to mobilize the superior tracheal segment, but these mobilizations were insufficient to perform an anastomosis. Thus, to allow additional mobilization, we performed a clamshell thoracotomy and incised the pericardium $360^{\circ}$ around the hilum, dissecting the pulmonary ligament bilaterally. These procedures allowed an anastomosis. An end-to-end bronchial anastomosis was carried out using running 4-0 PDS II sutures (Ethicon, Somerville, N.J., USA) for the membranous portion and interrupted 4-0 PDS II sutures for the cartilaginous portion. The bronchial anastomosis was wrapped with an omental pedicle flap that was lifted up through a retrosternal route via a laparotomy. A gastrostomy was also performed for postoperative enteral nutrition. For management after surgery, a chin suture was placed. Neck mobilization was restricted for the placement of the chin suture and airway fixation. After confirming an improvement in the laryngeal edema using a laryngoscopy, extubation was performed on postoperative day (POD) 17. The patient exhibited swallowing disturbances and underwent swallowing rehabilitation. Enteral nutrition was provided through the gastrostomy. The patient was discharged on POD 31, and per os feeding was started on POD 51. Radiotherapy (60 Gy in 40 daily fractions of $1.5 \mathrm{~Gy}$ ) was performed as an adjuvant therapy. Throughout the clinical course, no major complications, including swallowing disturbances, hoarseness or anastomotic problems, were observed. A histopathological examination of the resected specimen revealed the characteristic morphology of CASTLE 
Nogami et al:: A Case of Carcinoma Showing Thymus-Like Differentiation with a Rapidly Lethal Course

(fig. 3a); immunohistochemistry was positive for CD5 (fig. 3b) but negative for thyroid transcription factor 1 (TTF-1; fig. 3c).

Eleven months after surgery, lower back pain and right leg numbness developed and led to gait inability. Multiple lung and bone recurrences were observed, but no local recurrence. In order to ease the back pain, palliative radiotherapy (35 Gy in 14 fractions) to the bone metastasis was administered. Twelve months after surgery, drainage of pleural effusion was performed. She died of pleural metastasis 14 months after the initial diagnosis of CASTLE.

\section{Discussion}

The histological appearance of CASTLE is similar to that of thymic carcinoma, lymphoepithelioma or SCC. However, the clinical outcomes of patients with CASTLE are better than those of patients with SCC $[3,5]$. Distinguishing CASTLE from anaplastic carcinoma or SCC of the thyroid is important, because CASTLE exhibits a different biological behavior and is associated with a different prognosis. These more aggressive carcinomas often affect older patients, and patients may exhibit obstructive symptoms caused by a rapidly enlarging thyroid mass, extensive local infiltration and early distant metastases. The median survival period of patients with these carcinomas is about 6 months, despite a variety of treatment modalities. The critical point for the diagnosis of CASTLE is a careful morphological examination of hematoxylin-eosin-stained sections. CASTLE is characterized by the absence of papillary or follicular differentiation, but foci of squamous differentiation are common $[6$, 7]. Immunohistochemically, the tumor cells of CASTLE are negative for TTF-1 but positive for CD5, which is a marker of thymic carcinoma and the only marker capable of distinguishing it from thyroid SCC [8].

The use of postoperative radiotherapy to decrease the possibility of local recurrence should be considered, because CASTLE often exhibits a tendency for local recurrence and has been reported to be radiosensitive [4,9]. The use of chemotherapy in some patients has been reported, but the response was variable. As the number of case reports is relatively small, the role of chemotherapy in the treatment of CASTLE should be further investigated $[5,10]$.

Although the optimal treatment strategy remains uncertain because of the rarity of CASTLE, surgery is considered to be the first therapeutic option [5, 11, 12]. We decided to perform a tracheostomy after a thyroidectomy combined with the resection of the trachea. We reconstructed the resulting defect using an end-to-end anastomosis to maintain the patient's quality of life, since the bilateral vocal functions had been intact before surgery. In cases with a long tracheal defect, such as the present case, suprahyoid release and dissection of bilateral pulmonary ligaments can allow an end-to-end anastomosis to be performed [13]. An omental pedicle flap was used to wrap the anastomosis with prophylactic intent and to reduce the risk of anastomotic complications that could lead to critical problems.

In conclusion, we successfully treated a case of CASTLE by resecting the thyroid and invaded structures, though the patient had a bad clinical outcome. This case indicates that aggressive resection of the lesion with preservation of physiological functions might result in a favorable quality of life, and that radiotherapy might be effective in CASTLE because there was no local recurrence. 


\begin{tabular}{l|l}
\hline \multicolumn{2}{l}{ Case Rep Oncol 2014;7:840-844 } \\
\hline DOI: 10.1159/000370306 & $\begin{array}{l}\text { C 2014 S. Karger AG, Basel } \\
\text { www.karger.com/cro }\end{array}$ \\
\hline
\end{tabular}

Nogami et al:: A Case of Carcinoma Showing Thymus-Like Differentiation with a Rapidly Lethal Course

\section{Disclosure Statement}

The authors have no conflict of interest to declare.

\section{References}

1 Miyauchi A, Kuma K, Matsuzuka F, Matsubayashi S, Kobayashi A, Tamai H, Katayama S: Intrathyroidal epithelial thymoma: an entity distinct from squamous cell carcinoma of the thyroid. World J Surg 1985;9:128-135.

2 Chan JK, Rosai J: Tumors of the neck showing thymic or related branchial pouch differentiation: a unifying concept. Hum Pathol 1991;22:349-367.

-3 Luo CM, Hsueh C, Chen TM: Extrathyroid carcinoma showing thymus-like differentiation (CASTLE) tumor a new case report and review of literature. Head Neck 2005;27:927-933.

4 Ito Y, Miyauchi A, Nakamura Y, Miya A, Kobayashi K, Kakudo K: Clinicopathologic significance of intrathyroidal epithelial thymoma/carcinoma showing thymus-like differentiation: a collaborative study with Member Institutes of The Japanese Society of Thyroid Surgery. Am J Clin Pathol 2007;127:230-236.

-5 Roka S, Kornek G, Schuller J, Ortmann E, Feichtinger J, Armbruster C: Carcinoma showing thymic-like elements - a rare malignancy of the thyroid gland. Br J Surg 2004;91:142-145.

6 Dorfman DM, Shahsafaei A, Miyauchi A: Immunohistochemical staining for bcl-2 and mcl-1 in intrathyroidal epithelial thymoma (ITET)/carcinoma showing thymus-like differentiation (CASTLE) and cervical thymic carcinoma. Mod Pathol 1998;11:989-994.

7 Berezowski K, Grimes MM, Gal A, Kornstein MJ: CD5 immunoreactivity of epithelial cells in thymic carcinoma and CASTLE using paraffin-embedded tissue. Am J Clin Pathol 1996;106:483-486.

-8 Sun T, Wang Z, Wang J, Wu Y, Li D, Ying H: Outcome of radical resection and postoperative radiotherapy for thyroid carcinoma showing thymus-like differentiation. World J Surg 2011;35:1840-1846.

-9 Piacentini MG, Romano F, De Fina S, Sartori P, Leone EB, Rubino B, Uggeri F: Carcinoma of the neck showing thymic-like elements (CASTLE): report of a case and review of the literature. Int J Surg Pathol 2006;14:171175.

10 Kusada N, Hara Y, Kobayashi S, Weihua T, Nakamura Y, Kakudo K, Yuasa H: A case of aggressive carcinoma showing thymus-like differentiation with distant metastases. Thyroid 2005;15:1383-1388.

11 Yamazaki M, Fujii S, Daiko H, Hayashi R, Ochiai A: Carcinoma showing thymus-like differentiation (CASTLE) with neuroendocrine differentiation. Pathol Int 2008;58:775-779.

-12 Alifano M, Boudaya MS, Dinu C, Kadiri H, Regnard JF: Carcinoma showing thymus-like elements invading the trachea. J Thorac Cardiovasc Surg 2006;132:191-192.

13 Mathisen DJ: Surgery of the trachea. Curr Probl Surg 1998;35:453-542.
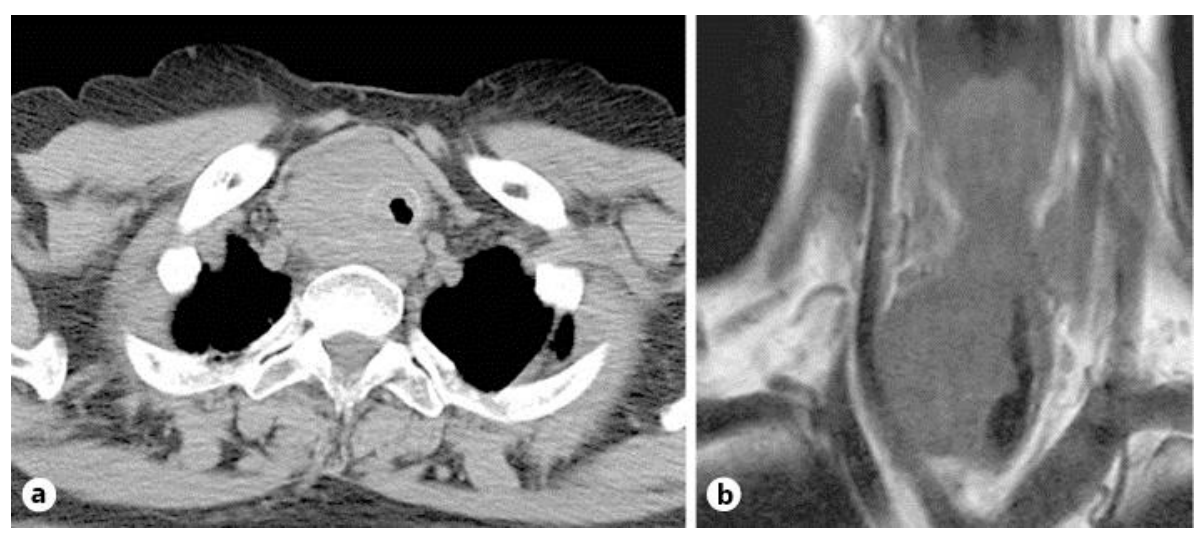

Fig. 1. a Cervical computed tomography image showing a thyroid tumor that has infiltrated the entire circumference of the trachea. b Magnetic resonance imaging showing a low possibility of esophageal invasion. 


\section{Case Reports in Oncology}

\begin{tabular}{l|l}
\hline \multicolumn{2}{l}{ Case Rep Oncol 2014;7:840-844 } \\
\hline DOI: 10.1159/000370306 & $\begin{array}{l}\text { C 2014 S. Karger AG, Basel } \\
\text { www.karger.com/cro }\end{array}$ \\
\hline
\end{tabular}

Nogami et al:: A Case of Carcinoma Showing Thymus-Like Differentiation with a Rapidly Lethal Course

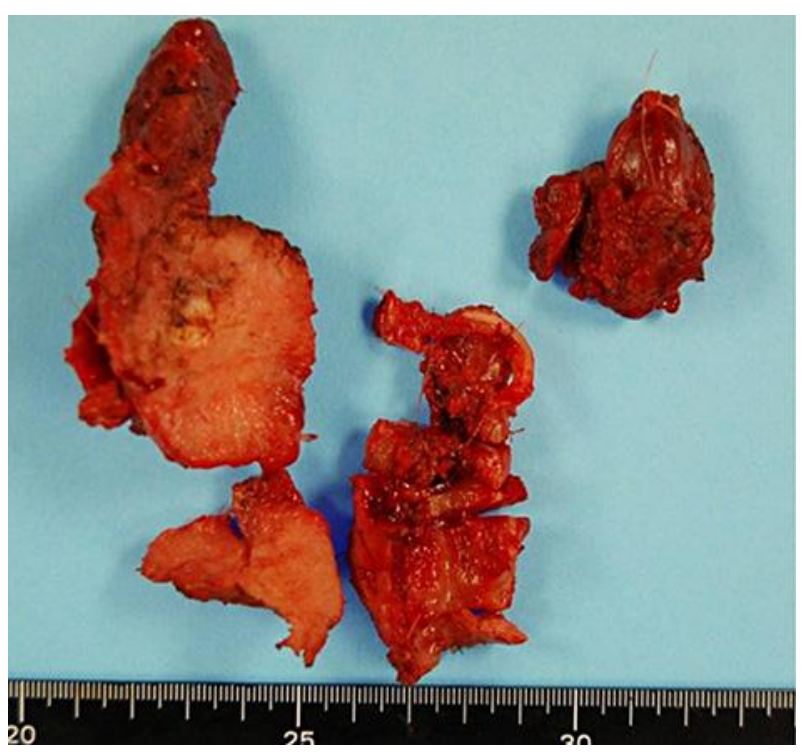

Fig. 2. Macroscopic examination showing the resected trachea (maximum size: $8 \times 6.5 \mathrm{~cm}$ ).
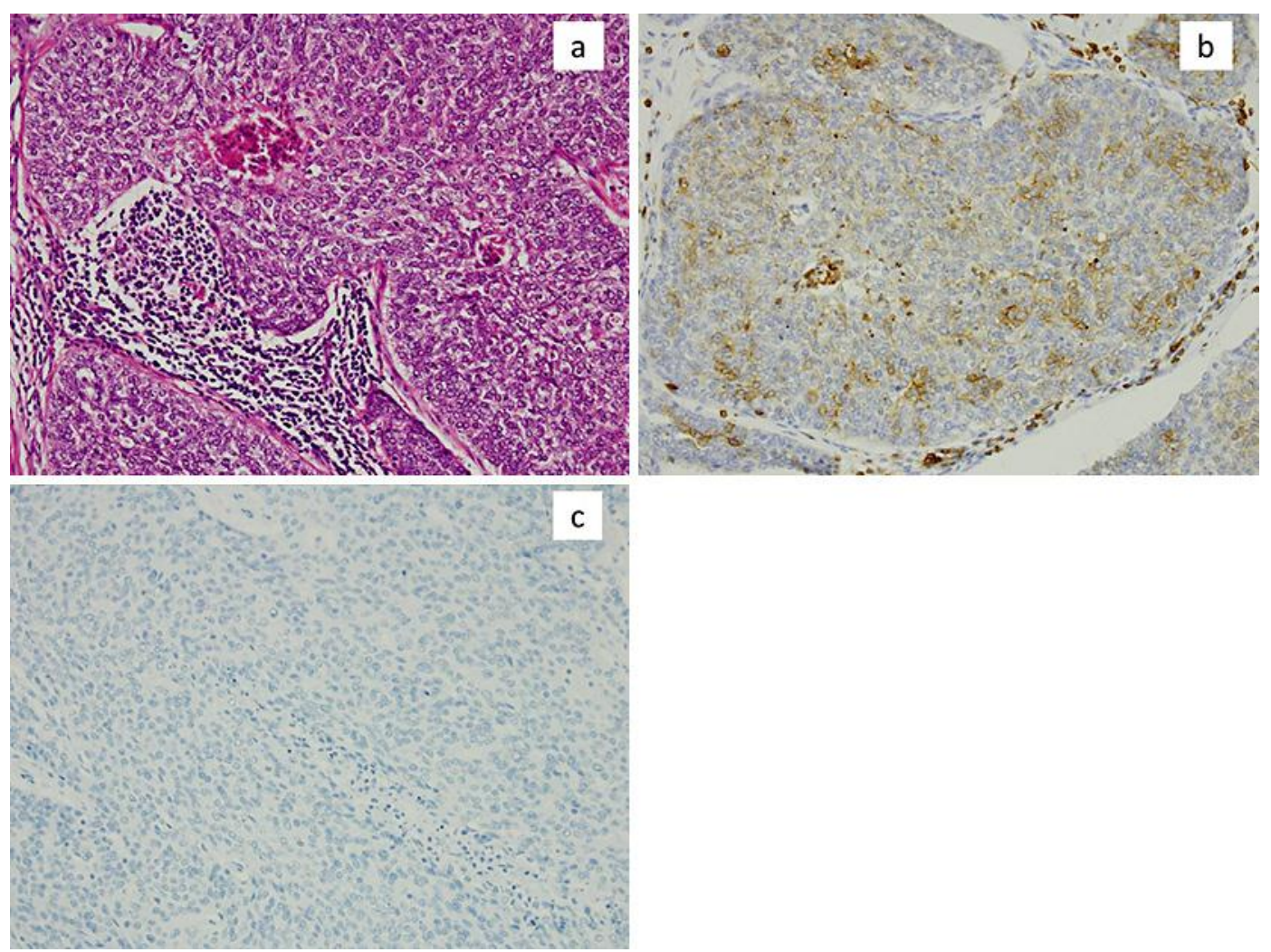

Fig. 3. Histopathological findings showing the separation of solid nests of squamous differentiated neoplastic cells, fibrous septa and the infiltration of numerous lymphocytes. a Low-power view by hematoxylin-eosin staining. b Immunostaining for CD5 revealed tumor cells with predominant membrane staining. c Immunostaining for TTF-1 revealed the absence of staining in the tumor cells. 